\title{
VENTRICULAR PARASYSTOLIC TACHYCARDIA
}

\author{
BY \\ KOO-YOUNG CHUNG,* THOMAS J. WALSH, $\dagger$ AND EDWARD MASSIE $\ddagger$
}

From the Department of Medicine, Washington University School of Medicine, and the Heart Station and Cardiovascular Laboratory, Barnes Hospital, St. Louis, Missouri, U.S.A.

Received July 30, 1964

Ventricular tachycardia like ventricular parasystole is rarely observed in the absence of cardiac disease (Massie and Walsh, 1960; Scherf and Bornemann, 1961), though several cases of functional ventricular tachycardia without demonstrable heart disease have been reported by Hair, Eagan, and Orgain (1962) and others. Parasystole consists of simultaneous activity of two independent impulse-forming centres, one of which is "protected" from the other, and each competing with the other to activate the atria and/or ventricles. The ectopic parasystolic pacemaker is commonly located in the ventricles but may be centred in the atria or atrio-ventricular node. Although many different investigators have reported various types of parasystole, only a few instances of ventricular tachycardia originating from a parasystolic focus have been published, the most recent examples being those of Scherf and Bornemann (1961) and Scherf, Blumenfeld, and Yildiz (1962).

In this paper, 11 cases of ventricular parasystolic tachycardia collected from the Barnes Hospital Heart Station will be presented.

\section{CASE REPORTS}

Case 1. B.N., a 74-year-old man, was admitted to Barnes Hospital because of cholelithiasis for which a cholecystectomy was performed. Cardiac symptoms or signs were absent, though his chest film disclosed minimal cardiomegaly. A routine electrocardiogram showed sinus rhythm with frequent ventricular parasystolic beats and brief periods of parasystolic tachycardia. There was no other abnormality though high voltage of the QRS complexes was present in lead V5 (Fig. 1). The sinus rate was 85 a minute and the ectopic rate 135 a minute. In one lead (lead II) a short period of ventricular parasystolic tachycardia without exit block was present. Several areas showed consecutive parasystolic beats with $2: 1$ or 3:1 exit block without direct interference from sinus beats. Occasional ventricular fusion beats (marked FB), characteristic of ventricular parasystole, were evident in leads aVR, V5, and V6. The shortest interectopic interval was between $40 \S$ and $47 \S$.

Case 2. A. M., a 39-year-old woman, was seen at the Washington University Clinics with an irregular pulse rate without cardiac symptoms or signs. The chest radiograph failed to show cardiomegaly. Numerous electrocardiograms on different occasions revealed the dominant rhythm of ventricular parasystolic tachycardia without any other abnormality (Fig. 2). The basic rhythm was sinus with a rate of 78 a minute. Occasional ventricular fusion beats were present. The direct interectopic interval varied between $74 \S$ and 88§. Some interectopic intervals in lead aVF (B) which contained sinus beats were longer than multiples of the basic interectopic interval. The parasystolic rate was 70 per minute.

* Fellow of National Institute of Health.

$\dagger$ Assistant Professor of Clinical Medicine.

$\ddagger$ Associate Professor of Clinical Medicine.

$\S$ These numbers represent hundredths of a second. 
Fig. 1.-Case 1. Sinus rhythm with intermittent ventricular parasystole and an area of ventricular parasystolic tachycardia (in lead II). There are occasional ventricular fusion beats (marked FB). The sinus rate is 85 a minute and the ectopic one is 135 a minute.

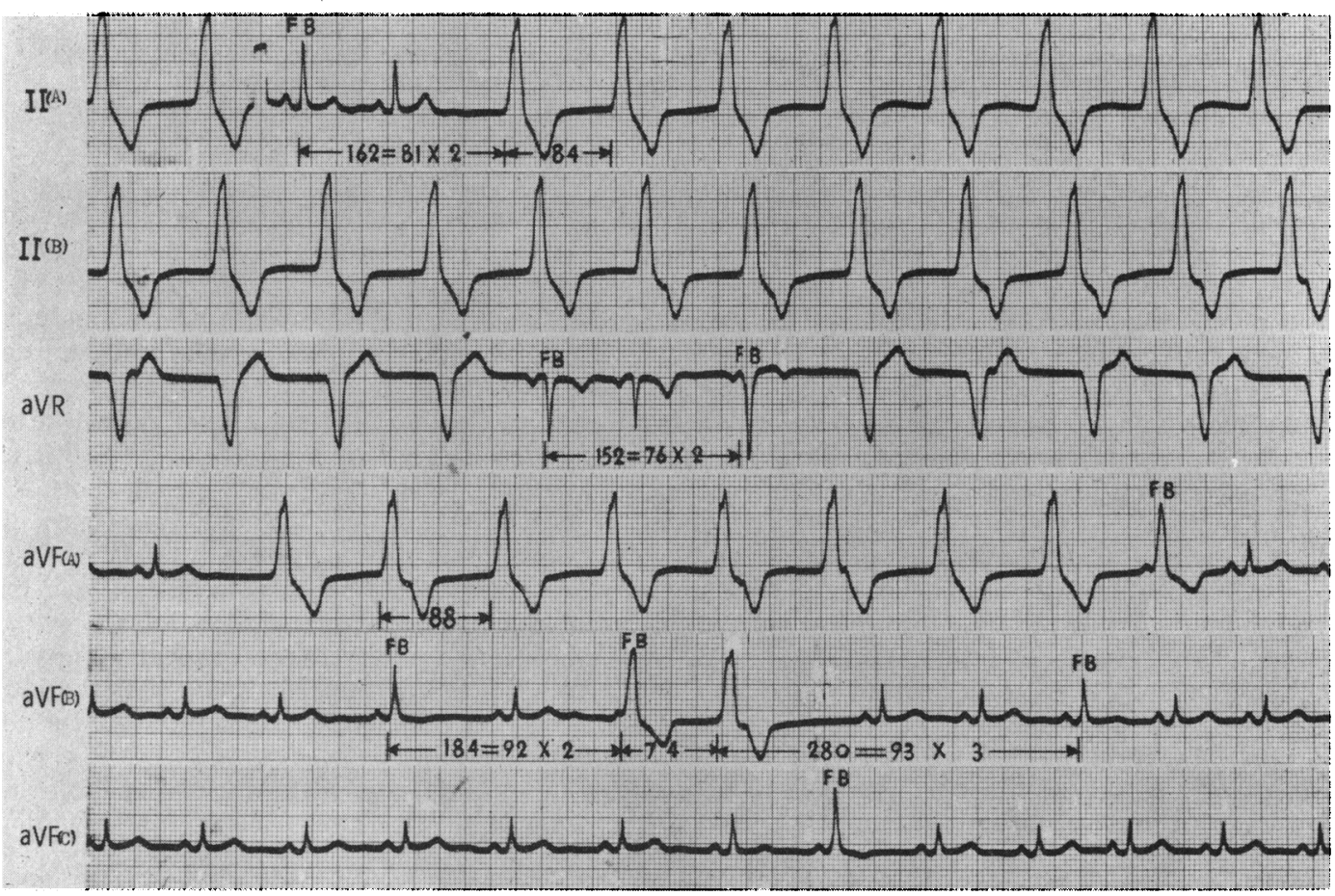

FIG. 2.-Case 2. Lead II (A) and (B) are continuous strips and lead aVF (A), (B), and (C) are also continuous. The tracing shows slow ventricular parasystolic tachycardia with intermittent sinus rhythm. There are occasional ventricular fusion beats (marked FB). The sinus rate is 78 a minute and the parasystolic rate is 70 a minute. 


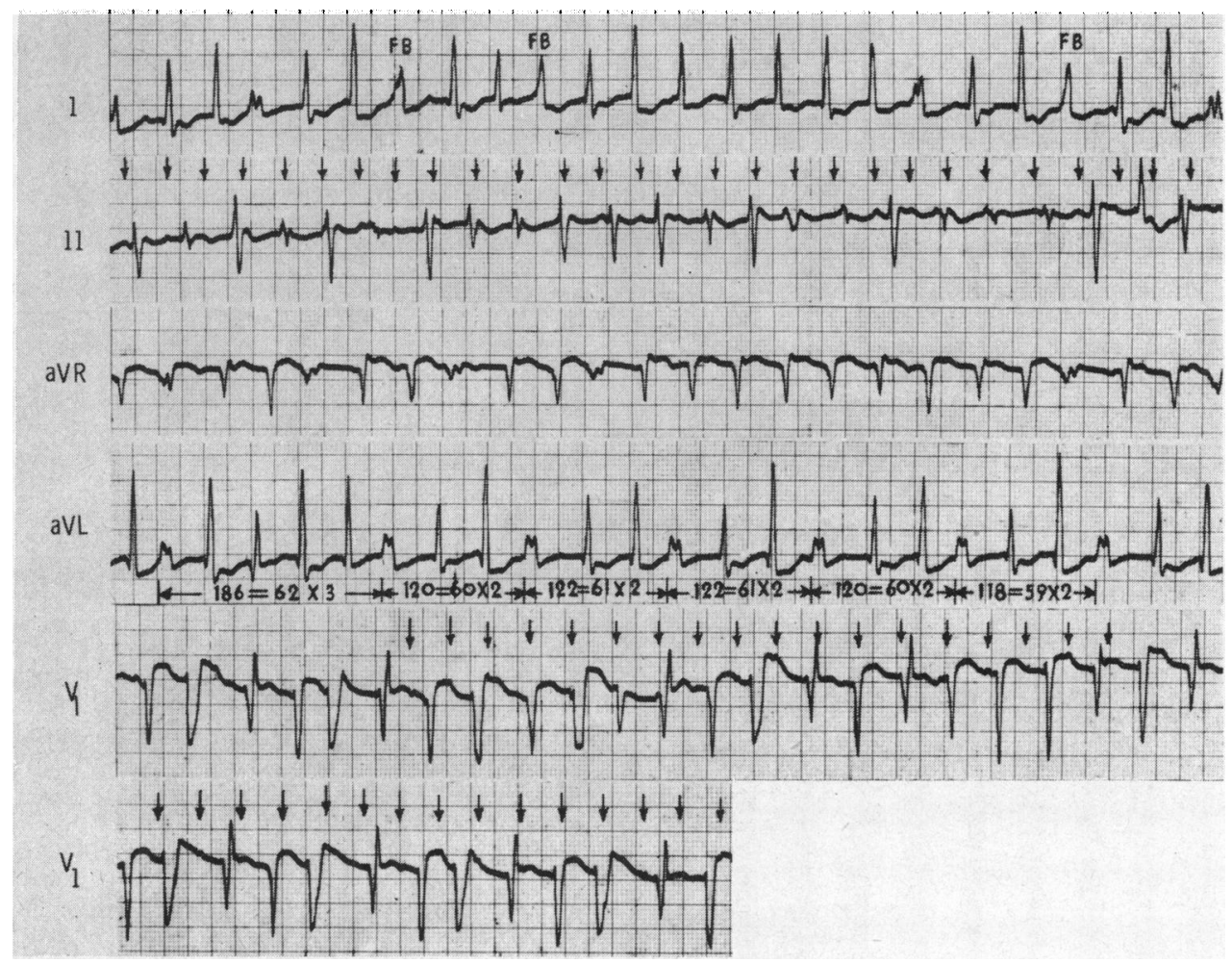

FIG. 3.-Case 3. Lower two strips of lead V1 are continuous. The tracing shows A-V nodal tachycardia and atrial tachycardia producing complete A-V dissociation and frequent ventricular parasystolic beats. The A-V nodal rate is 150 a minute, atrial rate 185 , and the ventricular parasystolic rate 95 a minute. There are occasional ventricular fusion beats (marked FB) in addition to aberrantly conducted ventricular beats originating from the A-V node. Most of the areas of ventricular tachycardia show $2: 1$ exit block.

Case 3. H. A., a 70-year-old man, was admitted to Barnes Hospital because of probable digitalis intoxication; he died of cerebral and myocardial infarctions three weeks after admission. The chest radiograph showed cardiomegaly, mainly left ventricular hypertrophy, and pleural effusion. An electrocardiogram on admission (Fig. 3) revealed an ectopic atrial tachycardia of 185 a minute and atrio-ventricular nodal tachycardia with the rate of 150 a minute producing complete A-V dissociation and frequent ventricular parasystolic beats. Digitalis intoxication was diagnosed, but the patient expired despite discontinuation of digitalis therapy and other therapeutic measures. In this figure, the shortest interectopic interval of the ventricular parasystole cannot be directly illustrated because of the presence of 2:1 or 3:1 exit block. There are frequently aberrantly conducted A-V nodal beats in addition to the bizarre QRS complexes of ventricular parasystolic beats. There are also many ventricular fusion beats between the A-V nodal and ventricular parasystolic beats. The shortest interectopic interval is between $59^{*}$ and $62^{*}$.

Case 4. S. R., a 54-year-old man who had had an anterior myocardial infarction three years previously, was readmitted to the Barnes Hospital because of a recent diaphragmatic myocardial infarction. He developed many episodes of ventricular tachycardia, originating from a parasystolic focus, for which large doses of procainamide (pronestyl) were repeatedly required. The patient continued to experience intermittent ventricular tachycardia despite the maintenance of this therapy though the frequency of the episodes became less. A chest film showed no cardiomegaly. Electrocardiograms, such as that in Fig. 4, on many occasions revealed ventricular parasystolic tachycardia with occasional sinus beats resembling the record of Fig. 2 except for the more rapid rate of 140 a minute. Occasional ventricular fusion beats are present.

* These numbers represent hundredths of a second. 


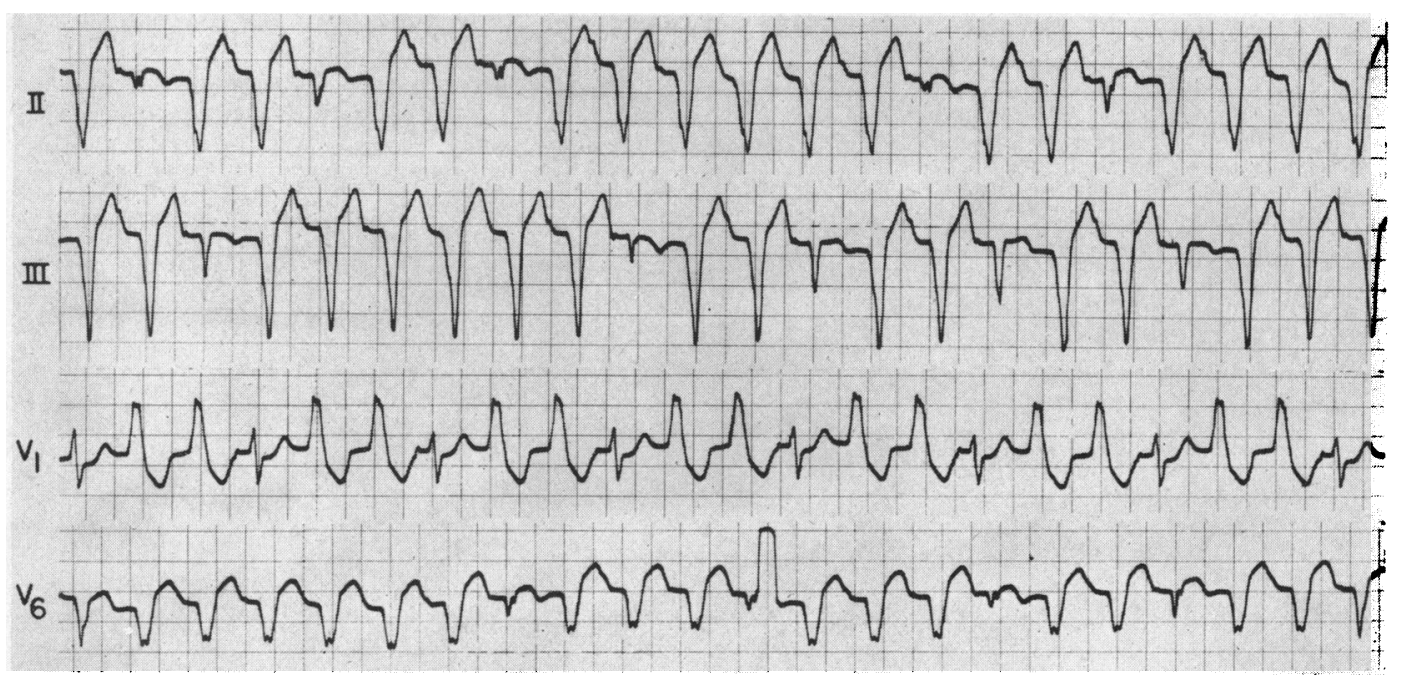

FIG. 4.-Case 4. Ventricular parasystolic tachycardia with rate of 140 a minute and occasional sinus beats. There are occasional ventricular fusion beats. [Reproduced from Clinical Vectorcardiography and Electrocardiography, by Edward Massie, M.D., and Thomas J. Walsh, M.D. (Fig. 468, p. 570), Chicago, 1960, The Year Book Publisher, Inc., with permission of publishers and authors.]

Direct measurement of the sinus cycle is not possible in this tracing. It is interesting to note that the ventricular parasystolic rhythm is the dominant rhythm. The parasystolic cycle is between $40^{*}$ and $44^{*}$.

Case 5. J. T., a 48-year-old man who had a previous history of myocardial infarction, was admitted to Barnes Hospital because of recurrent chest pain. A chest radiograph showed left ventricular hypertrophy.

His electrocardiogram indicated an old diaphragmatic - posterior myocardial infarction which was also confirmed by vectorcardiography. Intermittent ventricular parasystolic tachycardia was found. In Fig. 5A, the coupling intervals of the parasystolic beats vary very much but the shortest interectopic intervals are relatively constant (varying between 0.49 and $0.54 \mathrm{sec}$.). The parasystolic rate is about 110 a minute.

Case 6. K. R., a 75-year-old man who had a history of past posterior myocardial infarction, was admitted to Barnes Hospital because of bronchopneumonia and expired a few days after admission. Several electrocardiograms similar to the one illustrated in Fig. 5B displayed atrial fibrillation with ventricular parasystolic tachycardia.

Case 7. J. W., a 52-year-old man who had had a myocardial infarction with a complicating cerebral thrombosis three years previously, came to the Physical Therapy Department of the Washington University Clinics for treatment of a residual left hemiplegia. His chest film showed minimal left ventricular hypertrophy. One of his electrocardiograms (Fig. 6A) demonstrated a sinus rhythm with intermittent ventricular parasystolic tachycardia with a rate of 115 a minute. The complete tracing revealed an old anterior myocardial infarction which was confirmed vectorcardiographically. The coupling intervals of the parasystolic beats (marked FB) were also evident.

Case 8. O. F., a 72-year-old man, was admitted to Barnes Hospital because of an acute myocardial infarction and expired two weeks after admission. An electrocardiogram on admission confirmed the acute anterior myocardial infarction and also demonstrated in lead II (Fig. 6B) an intermittent ventricular tachycardia.

Case 9. K. F., a 69-year-old man, who had had a left pneumonectomy for carcinoma of the lung and a transverse colostomy for sigmoid volvulus, was admitted to Barnes Hospital in shock and expired a few days after admission because of bowel obstruction resulting from old surgical adhesions and congestive heart failure. He had a long history of atrial fibrillation due to arteriosclerotic heart disease. One of his electrocardiograms showed atrial fibrillation with intermittent ventricular parasystolic tachycardia (Fig. 7).

Case 10. S. J., an 86-year-old man who had a history of an old diaphragmatic myocardial infarction with intermittent congestive heart failure and bronchogenic carcinoma of the lung was admitted to Barnes Hospital because of lobar pneumonia. A chest radiograph revealed evidence of bronchogenic carcinoma, * These numbers represent hundredths of a second. 


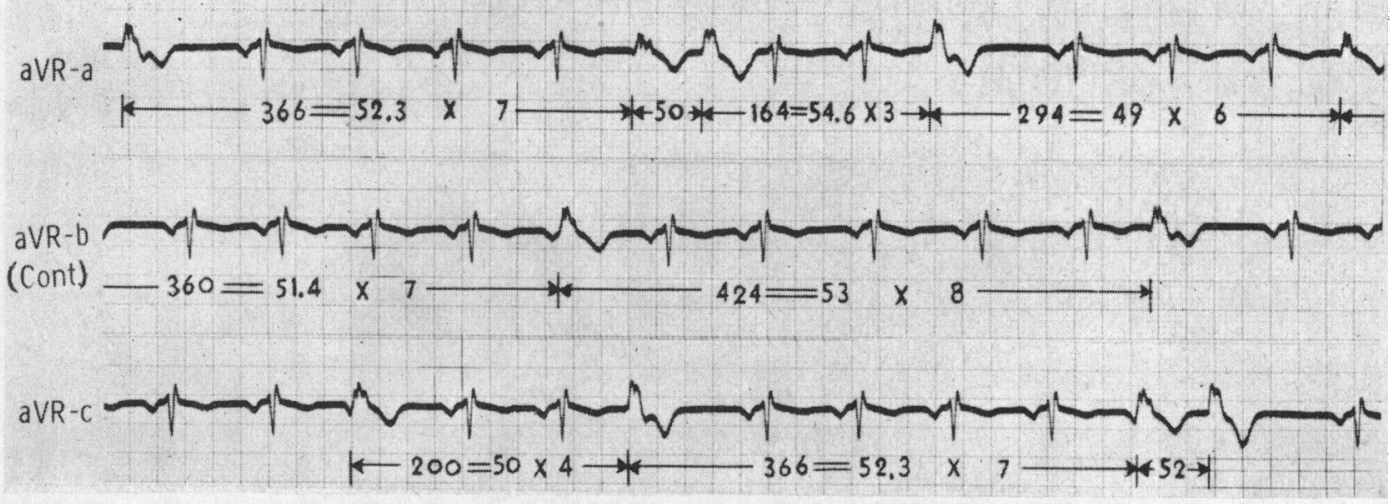

A

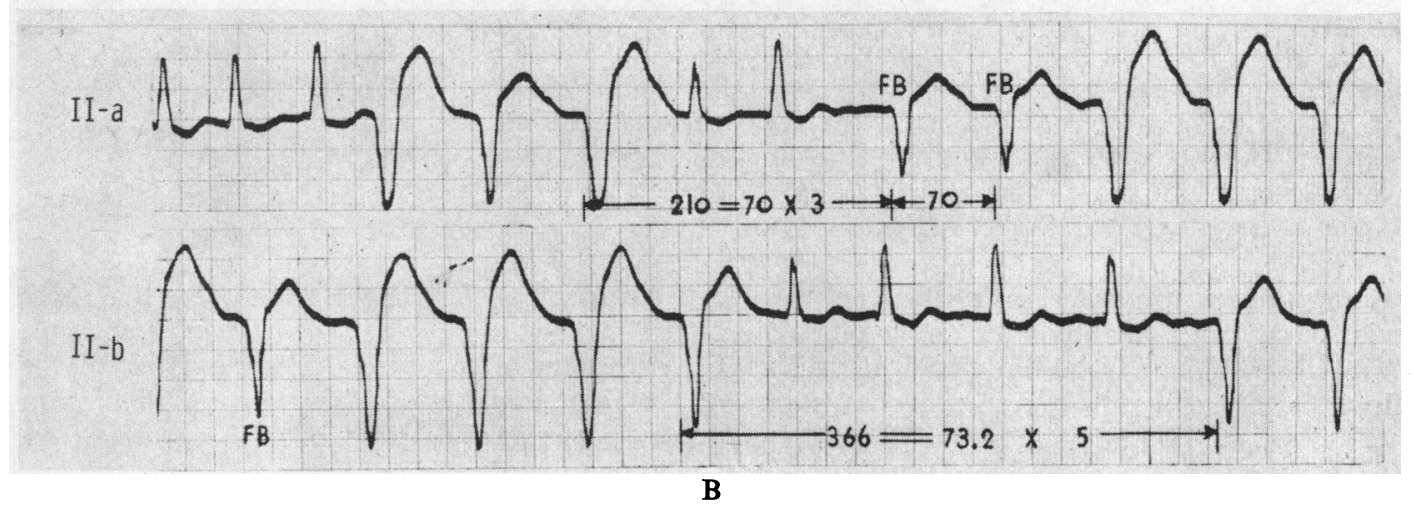

FIG. 5A.-Case 5. Leads aVR-a and b are continuous. The basic rhythm is sinus with intermittent ventricular parasystolic tachycardia with a rate of 110 a minute.

FIG. 5B.-Case 6. Leads II-a and $b$ are not continuous. The tracing shows atrial fibrillation with intermittent ventricular parasystolic tachycardia. The rate of the basic rhythm is 100 a minute and of the parasystolic rhythm 80 a minute. Note there are occasional ventricular fusion beats (marked FB).

emphysema, and left ventricular hypertrophy. A routine electrocardiogram (Fig. 8) was interpreted as sinus rhythm with intermittent ventricular parasystolic tachycardia.

Case 11. J. M., a 38-year-old physician, was referred to our medical centre for evaluation of an arrhythmia associated with occasional palpitation. No evidence of cardiac disease was found clinically or by laboratory tests except for the abnormal cardiac rhythm. His electrocardiogram (Fig. 9) disclosed ventricular parasystolic tachycardia and intermittent sinus tachycardia. The parasystolic tachycardia became all the more predominant with physical exercise or vagal stimulation.

\section{Results AND COMMENT}

Ventricular tachycardias originating from a parasystolic focus have been induced experimentally by various authors (Scherf et al., 1962; Scherf and Chick, 1951) and have also been observed clinically. The above authors produced experimental ventricular parasystolic tachycardia with - rates of 150 to 170 a minute in dogs using sodium chloride or sodium citrate or oxalate. Scherf's group has also reported 8 patients with ventricular parasystolic tachycardia with rates between 86 and 150 a minute. In our series, the ventricular parasystolic tachycardia rates varied between 70 and 140 a minute. The 11 cases in this study are described in the Table in relation to their clinical features and other pertinent electrocardiographic findings. 


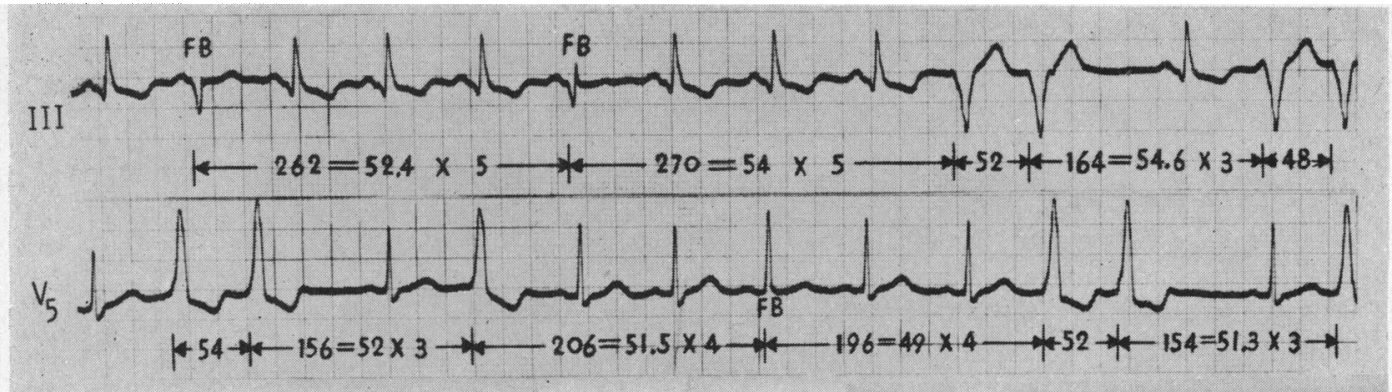

A

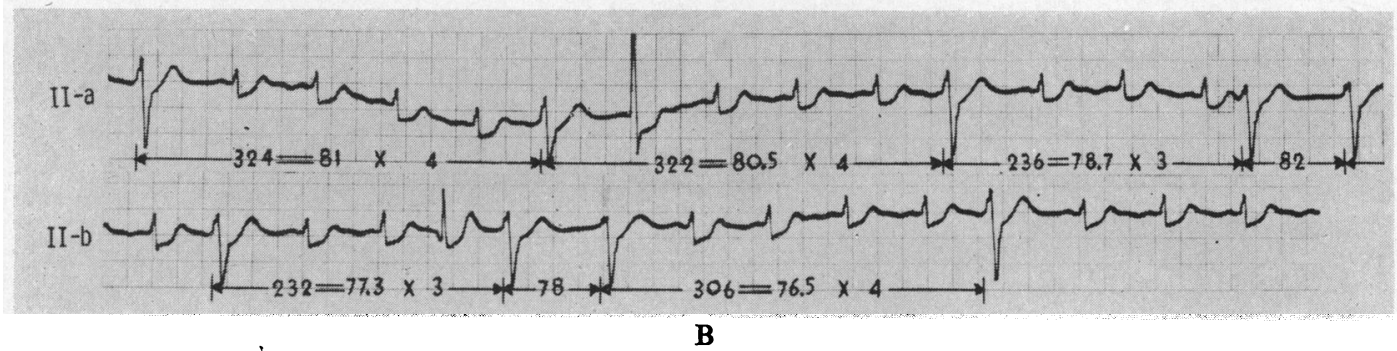

Fig. 6A.-Case 7. Sinus rhythm with intermittent ventricular parasystolic tachycardia with a rate of 115 a minute. Note three ventricular fusion beats (marked FB).

FIG. 6B.-Case 8. Leads II-a and $b$ are continuous. The tracing shows sinus rhythm with frequent ventricular parasystolic beats and one nodal escape beat (the 7th beat of lead II-a). The last portion of lead II-a and mid-portion of lead II-b show two successive ventricular parasystolic beats and other areas exhibit $3: 1$ or $4: 1$ exit block in parasystolic tachycardia. The shortest interectopic intervals vary between 0.76 and 0.81 second.
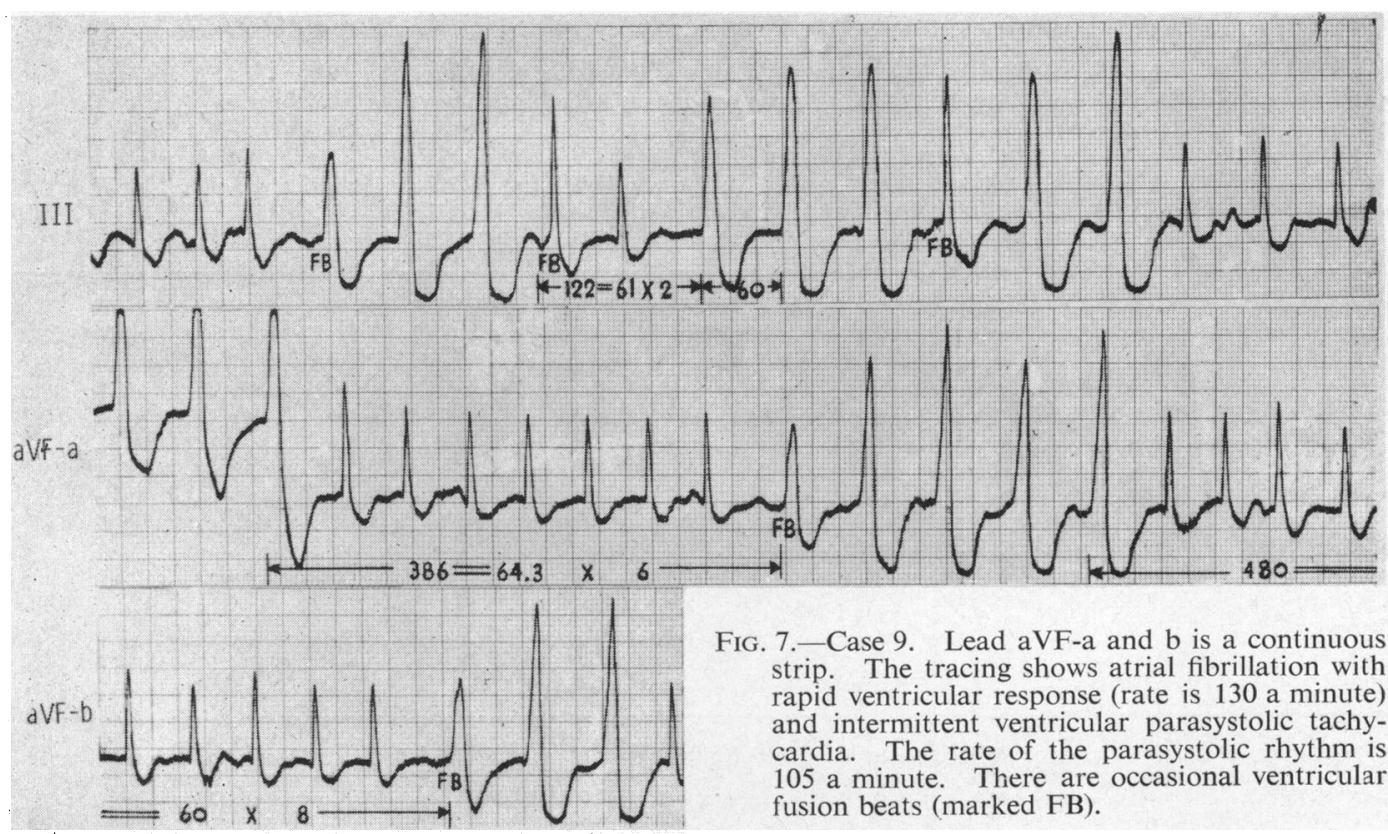

FIG. 7.-Case 9. Lead aVF-a and b is a continuous strip. The tracing shows atrial fibrillation with rapid ventricular response (rate is 130 a minute) and intermittent ventricular parasystolic tachycardia. The rate of the parasystolic rhythm is 105 a minute. There are occasional ventricular fusion beats (marked FB). 
It is noted that 2 patients in our series (Cases 2 and 11) did not show any evidence of organic heart disease or cardiomegaly though all those reported by Scherf and Bornemann (1961) had organic heart disease. These two presented a rather slow ventricular parasystolic tachycardia of at

TABLE

An Analysis of 11 Cases of Ventricular Parasystolic Tachycardia in Relation to their Clinical Features AND OTHER ELECTROCARDIOGRAPHIC FINDINGS

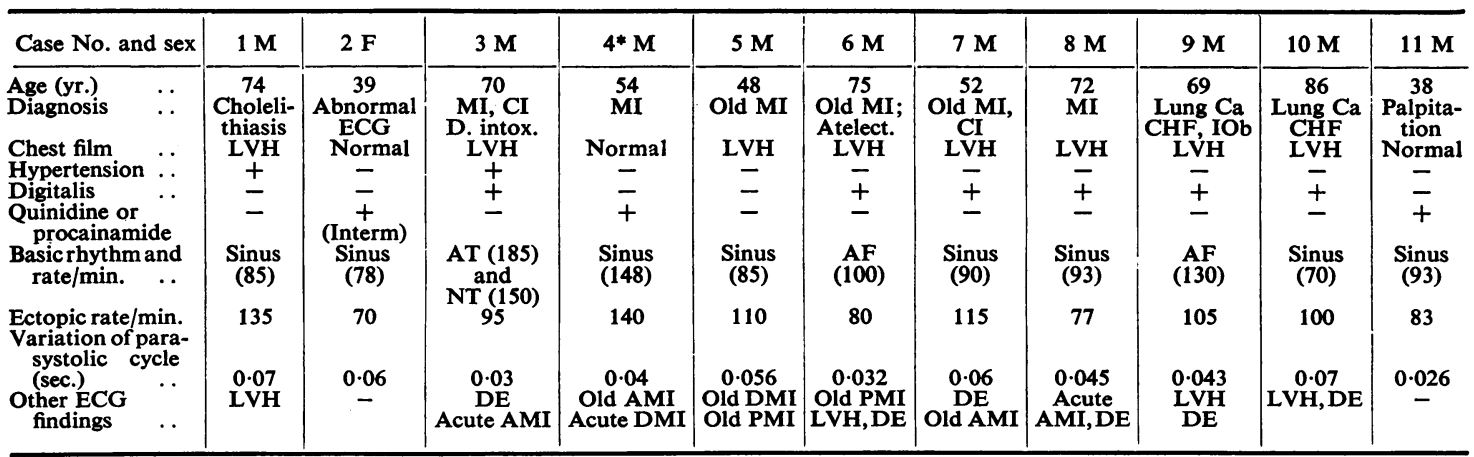

LVH, left ventricular hypertrophy; AMI, anterior myocardial infarction; MI, myocardial infarction; DMI, diaphragmatic myocardial infarction; CI, cerebral infarction; PMI, posterior myocardial infarction; Interm, intermittent; Atelect., atelectasis of lung; AT, atrial tachycardia; Ca, cancer; NT, nodal tachycardia; CHF, congestive heart failure; AF, atrial fibrillation; 1Ob, intestinal obstruction; DE, digitalis

effect: D. intox., digitalis intoxication.
*This case has previously been presented in electrocardiographic form in Clinical Vectorcardiography and Electrocardiography, by Edward Massie, M.D. and Thomas J. Walsh, M.D., Chicago, 1960. The Year Book Publishers, Inc. 


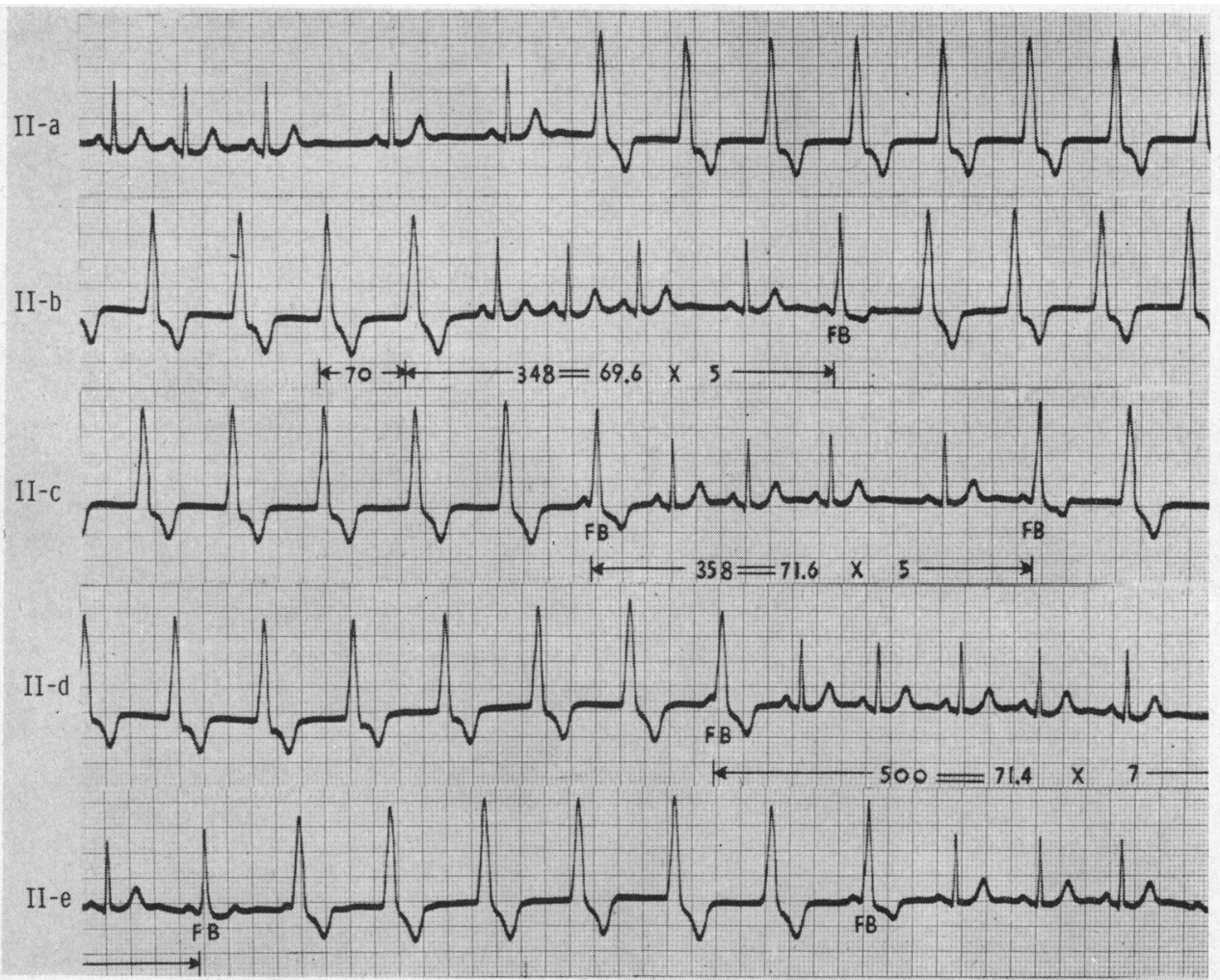

FIG. 9.-Case 11. Leads II-a, b, c, d, and e are continuous. The record shows predominant ventricular parasystolic tachycardia with a rate of 83 a minute and intermittent sinus rhythm with a rate of 93 a minute. It is interesting to note that the ectopic beats appear whenever the sinus rate slows. There are occasional ventricular fusion beats (marked FB).

least several years' duration. In them, the sinus rate was slightly faster than the parasystolic rate but the latter rhythm was the dominant one. They did not receive continuous treatment for the arrhythmia because they failed to reveal any abnormal cardiac symptoms or signs and the ectopic rate was approximately the same as the average normal sinus rate. On several occasions, quinidine was given and the parasystolic rhythm responded promptly to this medication. Another interesting finding in Case 2 is that the ectopic tachycardia disappeared completely with exercise when the sinus rate became more rapid, up to 100 to 120 a minute, and the ectopic beats did not reappear for some time even after the sinus rate returned to the resting level of 78 a minute. Carotid pressure was applied on several occasions without any effect on the arrhythmia. In contrast to this finding, the other patient (Case 11) developed the parasystolic tachycardia during and after exercise when the sinus rate became faster and also during vagal stimulation, an experience similar to that of Scherf and his associates in several reports (Scherf and Chick, 1951; Scherf and Schott, 1953; Scherf et al., 1957; Scherf and Bornemann, 1961). This finding (vagal stimulation effect on parasystole) was first described by Vedoya (1944) and Vedoya, Dumas, and Urdapilleta (1948) in a patient with atrioventricular nodal rhythm. In addition, carotid pressure may slow (Scherf and Bornemann, 1961; Golbey et al., 1954) or provoke the parasystole (Mueller and Baron, 1953). On the other hand, disappearance of the parasystole has been reported due to the same mechanism.

One patient of our study (Case 3) presented a very interesting triple tachycardia, that is, he had double supraventricular (paroxysmal atrial tachycardia and atrio-ventricular nodal) tachycardia with a ventricular rate of 150 a minute producing complete atrio-ventricular dissociation and a 
ventricular tachycardia originating from a parasystolic focus with a rate of 95 a minute. This triple tachycardia was thought to be due to digitalis excess; a similar case was reported by Katz and Pick (1956). Occasional ventricular fusion beats and aberrantly conducted ventricular beats originated from the atrio-ventricular node probably as a consequence of the rapid nodal tachycardia. The coupling intervals in this case did not vary conspicuously since the rates of the basic rhythms, i.e. the atrio-ventricular nodal tachycardia and ventricular tachycardia, were relatively fast. This phenomenon (the relative constancy of the coupling intervals) was also observed in Case 4 of our study and encountered as well in the experimental study reported by Scherf et al. (1962).

In Case 4, the calculated ectopic periods in the pauses filled with sinus beats were occasionally slightly shorter than the ectopic period. This experience is a well-known phenomenon (Faltitschek and Scherf, 1932; Scherf and Schott, 1953), seen clinically (Holzmann, 1957) and experimentally (Scherf et al., 1962), but not fully explained. This finding, however, is not always present (Scherf and Chick, 1951) in ventricular parasystolic tachycardia and the opposite relationship may occur with this arrhythmia (see Fig. 2 and 9). In rapid ventricular parasystolic tachycardia (Case 4), the protection of the centre may be due to the rapidity of the impulse formation rather than to a hypothetical block surrounding the ectopic focus. This phenomenon has been observed in an experimental study by Scherf et al. (1962). However, it is worth noting that, on the average, the rates of ventricular parasystolic tachycardias are slower than those of paroxysmal ventricular tachycardia without the parasystolic mechanism (Massie and Walsh, 1960; Katz and Pick, 1956).

All patients except one in our series were men. With the exception of the two youngest (Cases 2 and 11), all the others had serious organic heart disease; 6 were older than 69 years; 3 patients had recent myocardial infarctions and 5 had older lesions; 2 had had at least two different coronary thromboses.

\section{SUMMARY}

Eleven cases of ventricular parasystolic tachycardia have been described: 9 patients showed evidence of organic heart disease and/or cardiomegaly and the 2 youngest failed to show signs of cardiac involvement. The ectopic rate varied between 70 and 140 a minute in parasystolic tachycardia. One patient showed a very unusual triple tachycardia, the ætiology of which was considered to be digitalis intoxication. Of the 11 patients, 7 had at least one myocardial infarction. In our experience the relatively slow ventricular tachycardias almost always originated from a parasystolic focus. Further follow-up study will be necessary to investigate the clinical significance of the occurrence of slow ventricular parasystolic tachycardia in young and otherwise healthy patients.

We gratefully acknowledge the able technical assistance of Mrs. Edna Comfort, Mrs. Shirley Gonzalez-Rubio, Miss Judy Schukar, Miss Sandra Steinhauser, and Mrs. Glenna Wissner.

\section{REFERENCES}

Faltitschek, F., and Scherf, D. (1932). Klinischer Beitrag zur Parasystoliefrage. Wien. Arch. inn. Med., $23,269$.

Golbey, M., Ladopulos, C. P., Roth, F. H., and Scherf, D. (1954). Changes of ventricular impulse formation during carotid pressure in man. Circulation, 10, 735.

Hair, T. E., Jr., Eagan, J. T., and Orgain, E. S. (1962). Paroxysmal ventricular tachycardia in the absence of demonstrable heart disease. Amer. J. Cardiol., 9, 209.

Holzmann, M. (1957). Zur Differentialdiagnose der Kammertachykardien. Cardiologia (Basel), $30,204$.

Katz, L. N., and Pick, A. (1956). Clinical Electrocardiography, Part 1. Lea and Febiger, Philadelphia.

Massie, E., and Walsh, T. J. (1960). Clinical Vectorcardiography and Electrocardiography. Year Book Publishers, Chicago.

Mueller, P., and Baron, B. (1953). Clinical studies on parasystole. Amer. Heart J., 45, 441.

Scherf, D., Blumenfeld, S., and Yildiz, M. (1962). Extrasystoles and parasystole. Amer. Heart J., $64,357$.

- and Bornemann, C. (1961). Parasystole with a rapid ventricular center. Amer. Heart J., 62, 320.

- , and Chick, F. B. (1951). Experimental parasystole. Amer. Heart J., 42, 212.

-, and Schott, A. (1953). Extrasystoles and Allied Arrhythmias. Heinemann, London.

-, , Reid, E. C., and Chamsai, D. G. (1957). Intermittent parasystole. Cardiologia (Basel), $30,217$.

Vedoya, R. (1944). Parasistolia. López, Buenos Aires.

-, Dumas, J. J., and Urdapilleta, V. (1948). Comentarios sabre dos casos de Parasistolia. Rev. argent. Cardiol., 15,364 . 